\title{
Por la idea del bien: múltiples miradas
}

\author{
Rómula Llanes-Barbuzano* \\ Esther Diviñó-González ${ }^{\star *}$
}

\footnotetext{
Doctora en Ciencias Filológicas. Profesora titular del Departamento Lengua y

Comunicación, Facultad de Español para

No Hispanohablantes, Universidad de La

Habana, Cuba.

Correo electrónico:

romula.llb@fenhi.uh.cu.

** Doctora en Ciencias Lingüísticas. Profesora Auxiliar del Departamento

Lengua y Comunicación, Facultad de Español para No Hispanohablantes,

Universidad de La Habana, Cuba.

Correo electrónico:

esther.dg@fenhi.uh.cu.
}

Recibido: 22 de mayo del 2017

Aprobado: 22 de agosto del 2017

Cómo citar este artículo: Llanes-Barbuzano, Rómula, y Esther Diviñó-González. "Por la idea del bien: múltiples miradas".

Rastros Rostros 19.35 (2017): 1-12.

Web. doi: https://doi.org/10.16925/2382-

4921.2017.35.04

\section{Resumen}

Introducción: el artículo se propone focalizar la diversidad de percepciones, representaciones, posiciones psicológicas y éticas, opiniones y puntos de vista que la idea del bien puede generar en una época que se globaliza a pasos agigantados. Las autoras consideran la clase-espacio público de mediación lingüística entre adultos no hispanohablantes que aprenden el español como lengua extranjera en Cuba. Metodología: para estudiar las manifestaciones de las actitudes valorativas en ese contexto, se ha aplicado un modelo conversacional que supera las tendencias unilaterales de influencia educativa. Resultados: la reflexión se ha comenzado con el análisis de los tipos de texto orales y escritos producidos en la dinámica comunicativa, lo que ha sido inseparable del abordaje de nociones complejas, de permanente interconexión y generación como las de identidad, interculturalidad, carácter replicativo de la palabra, textualidad, heteroglosia, inter y transtextualidad del discurso. Conclusión: se reflexiona acerca la emergencia de las voces y valores del otro como axioma fundamental que sustenta la actitud intercultural del que aprende acerca de la idea del bien en un aula pluricultural.

Palabras clave: dialogismo, heteroglosia, idea del bien, sentido, significado. 


\title{
For the idea of good: multiple looks
}

\begin{abstract}
Introduction: the article aims to focus on the diversity of perceptions, representations, psychological and ethical positions, opinions and points of view that the idea of good can generate in an era that is globalizing at a rapid pace. The authors consider the class-public space of linguistic mediation between non-Spanish-speaking adults who learn Spanish as a foreign language in Cuba. Methodology: to study the manifestations of the evaluative attitudes in that context, a conversational model has been applied that overcomes the unilateral tendencies of educational influence. Results: the reflection has begun with the analysis of the types of oral and written texts produced in the communicative dynamic, which has been inseparable from the approach of complex notions, of permanent interconnection and generation as those of identity, interculturality, replicative character of the word, textuality, heteroglossia, inter and transtextuality of discourse. Conclusion: we reflect on the emergence of the voices and values of the other as a fundamental axiom that sustains the intercultural attitude of those who learn about the idea of good in a pluricultural classroom.
\end{abstract}

Key words: dialogism, heteroglossia, idea of good, meaning, meaning.

\section{Para a ideia do bem: vários looks}

\section{Resumo}

Introdução: o artigo objetiva enfocar a diversidade de percepções, representações, posicionamentos psicológicos e éticos, opiniões e pontos de vista que a ideia de bem pode gerar em uma época que está se globalizando em ritmo acelerado. Os autores consideram o espaço público de classe de mediação lingüística entre adultos não falantes de espanhol que aprendem espanhol como língua estrangeira em Cuba. Metodologia: para estudar as manifestações das atitudes avaliativas nesse contexto, aplicou-se um modelo conversacional que supera as tendências unilaterais da influência educacional. Resultados: reflexão começou com a análise dos tipos de texto oral e escritos produzidos na dinâmica comunicativa, que tem sido inseparável abordar noções complexas, interligação permanente e geração como identidade, multiculturalismo, caráter replicativo a palavra, textualidade, heteroglossia, inter e transtextualidade do discurso. Conclusão: refletimos sobre o surgimento das vozes e valores do outro como um axioma fundamental que sustenta a atitude intercultural de quem aprende sobre a ideia do bem em uma sala de aula pluricultural.

Palavras-chave: dialogismo, heteroglossia, ideia de bem, significado, significado. 


\section{Introducción $^{1}$}

Las nuevas posibilidades de comunicación que brindan los estudios sobre la idea del bien en un diálogo abierto con la del mal constituyen una interesante y polémica temática en el ámbito investigativo actual. El artículo se propone analizar algunas de las manifestaciones de la diversidad de percepciones, interpretaciones, opiniones, valores identitarios y éticos que la idea del bien significan en una época que se globaliza a pasos agigantados. Toda vez que esta constituye una representación, estará siempre mediado por el hombre. En contraste, este se siente cada vez más aislado, y el concepto de diversidad cultural todavía se proyecta en el sentido de considerar este fenómeno desde ángulos y perspectivas que promueven un posicionamiento de su mediador, el hombre contemporáneo, atrapado por la irracionalidad que emana directamente de la pluriculturalidad. ¡Nada más alejado de la realidad!

Hay que considerar que "[...] la cultura por condición natural varía; es decir, rasgos como costumbres, valores, normas, tipos de discursos van a cambiar en lo que se refiere a sus contenidos de un grupo humano a otro" (Austin 5) y que "[...] la asunción y orientación hacia un absoluto entendimiento mutuo plasmado en planes y estrategias, por muy bien elaborados que estén, no puede hacer que desaparezca la diversidad cultural en el futuro" (Lewis 21). Teniendo en cuenta esto, en este trabajo, se privilegia un concepto que posiciona al hombre no solo como sistema cognitivo que recibe un input para convertirlo en output, sino también como mediador, valor supremo que se forma en la interacción con el otro en cuanto este último puede tener una idea diferente sobre bien y el mal.

Teóricamente se parte de las contribuciones del enfoque histórico cultural que posiciona el lenguaje como actividad dialógica en la vida social (Leontiev; Bajtín, Estética de la creación verbal; Bajtín, Hacia una filosofía del acto ético; Bajtín, Marxismo y filosofía del lenguaje; Vigotsky, Pensamiento y Lenguaje; Vigotsky, La formación social de la mente), del condicionamiento cultural del pensamiento (Lewis), de

1 Resultado de dos proyectos de investigación inscritos en la Universidad de La Habana, Cuba: Sentido y significado en la heteroglosia: un proyecto para el occidente de Cuba. Desarrollo de las relaciones interculturales y el tratamiento de los valores en los cursos de la FENHI. la textualidad (Beaugrande), de la intertextualidad (Kristeva) y del análisis del discurso (Van Dijk, "Semántica del discurso e ideología", El discurso como estructura y proceso, Ideology, a Multidisciplinary Approach). Los estudios semántico-discursivos cubanos constituyen los referentes inmediatos (Caballero, "Estrategia, modalidad e ilucución", "Modalidades semánticas del lenguaje”, Semántica y diccionario; Curbeira, Lecturas desemántica, Introducción a la teoría del lenguaje, "Teoría y práctica del grupo de estudios semánticos", "La interacción de las ideologías y las categorías semánticas”; Galbán, "Aproximación al estudio de las macrocategorías semánticas modales", Wong, "Primer acercamiento al estudio de la macro-categoría", Un modelo para el análisis semántico discursivo de la causalidad).

A partir del postulado bajtiniano sobre el carácter replicativo de la palabra, la emergencia de las voces y valores del otro - comprendido no como lo opuesto, sino como parte de la heteroglosia constitutiva del lenguaje ${ }^{2}$ - deviene un axioma fundamental que devela el entendimiento, la comprensión, la interpretación y la valoración de lo dicho / escrito fenómenos todos sujetos no solo a la diversidad cultural, sino también a las vivencias personales - como propuestas de las nuevas posibilidades de comunicación que se presentan en un contexto pluricultural de cualquier comunidad humana, aunque se hable una misma lengua y, que se pone de manifiesto, de manera muy especial en las interacciones que se establecen en los cursos de corta duración (CCD) de la Facultad de Español para No Hispanohablantes (FENHI) de la Universidad de La Habana (UH).

\section{La idea del bien en la diversidad cultural}

Si a la idea del bien que se defiende se le supone un modo específico de representarse la realidad, la convivencia humana y el modelo de sociedad al que aspiramos, no podrá desvincularse de la noción de cultura en cuanto proceso de una mezcla de legados y costumbres que se forman entre experiencias históricas diversas, todas ellas emergentes, mutantes, contradictorias y por eso mismo legítimas (Juliano).

\footnotetext{
2 Toma en cuenta la diversidad de voces y valores como algo inseparable del discurso y, por tanto, del texto como polifonía.
} 
En condiciones de multiculturalidad, ello se aprecia mucho más porque

[...] normalmente los elementos que constituyen la cultura de un grupo social o pueblo pasan inadvertidos entre los miembros del mismo grupo. Pero algunos elementos, los que son distintivos, se ponen de relieve cuando dos o más grupos entran en contacto. (Sánchez 308).

Luego, la exploración de las riquezas y beneficios que puede aportar la diversidad cultural tanto en términos de información como de actividades, experiencias sostenibles y enriquecedoras no solo se manifiesta en el sentido de los conocimientos utilitarios, sino también de la subjetividad y la intencionalidad (normas de comportamiento, sentimientos, emociones, estados de ánimo) ante los objetos y lo dicho sobre la base de normas sociales que toman en cuenta el valor de los objetos y fenómenos de la realidad, pues "[...] solo lo que adquiere valor social puede tomar forma y dejar raíces" (Bajtín, Marxismo y filosofía del lenguaje 44).

A esto se debe el interés de las autoras por intercambiar reflexiones y experiencias en torno a esta temática tan interesante, pero al mismo tiempo compleja, ya que se adentra en la comunicación pluricultural e intercultural, y la manifestación de los valores identitarios y éticos en el discurso de adultos no hispanohablantes que han coincidido en un aula para aprender español en Cuba.

Por su propia naturaleza, la razón de ser de ese proceso está en la sensibilidad para entender y atender la emergencia de los reclamos del otro, cuya interpretación revela y permite la interiorización de significados y sentidos, que en cada una de las comunidades presentes en la clase de español como lengua extranjera tiene determinadas peculiaridades por su modo de segmentar la realidad y de dar mayor o menor relevancia a determinados rasgos. Tal relevancia se muestra en la manera de proyectar las distintas posiciones éticas y psicológicas que se asumen ante lo dicho, lo que incide en la valoración en cuanto macrocategoría semántico modal por excelencia.

En aras de asegurar el cumplimiento de los objetivos del proceso de enseñanza aprendizaje desde la perspectiva del estudiante como agente social, hablante intercultural y aprendiente autónomo, es necesario implementar cambios cualitativos en la intervención docente educativa, la cual, a partir del diagnóstico sistemático y progresivo de los problemas, incorpora nuevos espacios de reflexión y debate constructivo como mecanismos e instrumentos importantes para la movilización de las fuerzas de resistencia y su transformación en factores impulsores ante los nuevos entornos emergentes.

Por esto, el punto de análisis del presente trabajo se fija referencialmente en aportes del enfoque histórico cultural, relacionados con la concepción del lenguaje con actividad en la vida social (Vygotsky "Pensamiento", Leontiev). Esto se ve en la teorización sobre la contribución y emergencia de los otros participantes del intercambio dialógico como parte de la relación (Bajtín, Marxismo y filosofía del lenguaje), el condicionamiento cultural del pensamiento (Lewis), así como en el encuentro textual e intertextual (Kristeva; van Dijk "Semántica", "El discurso", "Ideology"). Esto en razón a que en el abordaje de la temática se compromete no solo la transmisión de los códigos y habilidades lingüísticas, sino su posicionamiento como centro de experiencias educativas en una perspectiva discursiva, lo cual tiene en cuenta la interdependencia de los disímiles factores que intervienen en el encuentro intersubjetivo e intertextual que se constituyen a partir del acceso a los dinamismos móviles y cambiantes de la otredad, esto sin perder de vista la integralidad humana y hacer posible su desarrollo y empoderamiento.

Desde estas consideraciones, se plantea la pregunta de cómo abordar uno de los aspectos que menos se tiene en cuenta en el proceso de lectura en el contexto de la diversidad cultural de los CCD de ELE: ¿en qué medida la interpretación de los sentidos valorativos expresados por parte de los estudiantes posibilita la expresión de la idea del bien a partir de opiniones, ideas y valores éticos e identitarios propios?

Se defiende la idea de que si en el aula se materializa "[...] la influencia de los factores, procesos y decisiones que tienen su origen en otros ámbitos espaciales e institucionales" (Coll 11), y si la esencia de las valoraciones radica en su capacidad de integrarse en el flujo de la actividad, mediándola desde un punto de vista semiótico (Llanes), será necesario asumirlas como un espacio privilegiado de indagaciones y sus aplicaciones psicopedagógicas como una eficaz influencia educativa en el aula.

La anterior hipótesis se sustenta en el hecho de que en el discurso quedan registradas tanto la tradición cultural de sujetos representantes de disímiles comunidades y sus peculiaridades de segmentar la realidad y dar relevancia a sus rasgos, así como también la diversidad de su desarrollo psicológico y 
de la concepción del mundo (Caballero, Semántica y diccionario). De esto se infiere que, en la valoración como macrocategoría semántico modal por excelencia (Galbán), se revelan acciones dinamizadoras de cada uno de los contenidos y actitudes que se pueden expresar ante lo dicho o escrito en las situaciones de multiculturalidad de los CCD, presididas por un objetivo educativo compartido: aprender español para comunicase en un medio hispanohablante. Esto contribuye a que los estudiantes puedan modelar su discurso con base en sus intenciones comunicativas, así como facilitar la comprensión de las intenciones del interlocutor, además de expresar y argumentar sus opiniones sobre lo dicho desde posiciones abiertas, participativas, no respaldadas por su posición social, ya que en el aula se propician relaciones sinérgicas y de igualdad.

Lo expresado se sustenta en el carácter abarcador que se le reconoce a la modalidad y, dentro ella, a la valoración; lo que muchas veces, no se toma suficientemente en cuenta en el proceso de enseñanza aprendizaje, en general y de una lengua extranjera, en particular.

\section{El proceso de comprensión y la valoración}

La comprensión de un texto de cualquier tipo y magnitud es una de las actividades esenciales en la formación de la idea del bien, pues constituye punto de partida para el desarrollo de un proceso tan complejo como la comunicación entre representantes de diversas culturas, pues hay que

[...] penetrar en el mundo socio-cultural del autor, asumir su posición para establecer las conexiones intertextuales, intratextuales y supratextuales. Ese proceso no es lineal: presenta pausas - para descifrar significados lexicales, fraseológicos y sintácticos-, retrocesos - para descifrar significados contextuales-y adelantos - para predecir o dar un salto en el texto (Acosta 78).

Como parte de ese proceso, es imprescindible considerar que una de características esenciales del hombre es la de valorar todo lo que forma parte de su entorno en los más diversos dominios de su existencia (Fabelo, Los valores y sus desafíos actuales, Práctica, conocimiento y valoración, La naturaleza del reflejo valorativo de la realidad). Esta opinión tiene implicaciones muy particulares, pues demanda consolidar un proyecto que haga de las relaciones entre sujetos el eje de la reflexión lingüística. Si en la reflexión se plantea la interacción verbal como realidad primera de la lengua, cuya cualidad inseparable es la valoración y se hace de tal planteamiento algo más que un principio inútil o una mera declaración de buenas intenciones, el análisis debe vertebrarse tomando en consideración el abordaje dimensional de los significados: referencial, ilocutivo-modal y gramático-constructivo.

En el caso concreto, en lo expresado por los estudiantes cuando se habla sobre la idea del bien a partir de la lectura, se reflejan las posiciones éticas y psicológicas ante lo escrito, lo que permite distinguir la base de las normas sociales que toman en cuenta el valor pragmático de los objetos y fenómenos que clasifican en una escala de valores axiológicos y paramétricos (Galbán).

Para lograrlo se han considerado los estudios realizados por una de las autoras (Diviñó, Estrategia linguodidáctica para el tratamiento), quien a los tres niveles de comprensión propuestos por Fique (1998), le ha añadido el de la valoración. En la figura 1 , se ha representado gráficamente la jerarquización de esos cuatro niveles.

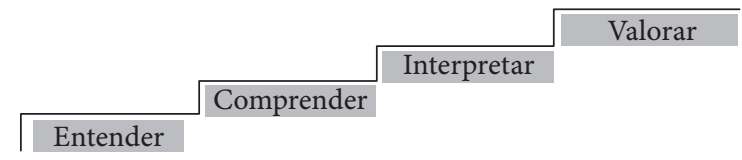

Figura 1. Niveles de comprensión de lectura. Fuente: Diviñó "Estrategia linguodidáctica" 2016.

Para obtener informaciones que permitan la realización de ese cuarto nivel en lo que a la idea del bien se refiere, una vía muy certera está en la determinación de la posición axiológica y los atributos modales del emisor. Las huellas que quedan registradas en el discurso permiten categorizar al emisor como: problemático/categórico, aprobador/desaprobador, tolerante/intolerante, reflexivo/irreflexivo, seguro/inseguro, interesado/desinteresado, sincero/insincero, atento/desatento y afectuoso/no afectuoso.

Una de esas huellas está dada por el contenido ilocutivo que puede manifestarse en una apreciación, una jerarquización o una evaluación. Se supone que la apreciación constituya la de más alta frecuencia en los CCD y que propicien una atmósfera favorable para la expresión de las opiniones en torno a lo que 
consideren bueno o malo. Por ello, el artículo la focaliza este tipo de valoración.

En ella, el emisor-receptor del discurso expresa o interpreta una intención comunicativa, en la que pone de manifiesto su posición subjetiva respecto a un objeto o a lo dicho por el otro sobre la base de una norma socializadora que no afecta al objeto valorado ni al sujeto valorador. Luego, no tiene que estar obligatoriamente respaldada por una posición social de institucionalidad del acto. Por lo tanto, tiene un carácter abierto, participativo, no transcendental y socialmente inofensivo. A pesar de esto, en el discurso sí quedan huellas de su posición social y de las relaciones de poder que se establecen.

Dado que los rasgos de dominio del objeto valorado: ético, estético, práctico utilitario, biológico, ideológico, emocional físico, intelectivo, entre otros, tienen un alto grado de universalidad desde el punto de vista categorial, pero que se realizan de manera diversa en las distintas culturas, se ha decidido abarcar solo el dominio ético psicológico por manifestar las normas de comportamiento, los sentimientos, las emociones y los estados de ánimos de los sujetos. Estos aspectos son muy importantes para lograr la interpretación de la emergencia de las voces y valores en la idea del bien. Para revelar las acciones dinamizadoras de cada una de las actitudes que se pueden expresar ante el contenido ilocutivo de lo dicho por el otro, se toma como punto de partida el carácter intertextual y transtextual de las ideas enunciadas por el otro.

\section{Evolución de las investigaciones en el campo de la integración de las valoraciones apreciativas ético psicológicas (VAE-P) en el aula universitaria}

El tema de la distinción, designación e intercambio de experiencias y significados en torno a la idea del bien a partir de la valoración de las cualidades de los objetos y de lo dicho por otros siempre ha sido una preocupación del hombre. A las cuestiones relacionadas con qué aceptar como bueno, justo, bello, útil, etc., o qué apreciar como lo opuesto el hombre les ha tenido que buscar respuestas para poder orientarse en las relaciones sociales en busca de fuerzas motivacionales que guíen su actividad y comportamiento.

El escenario socioeconómico nacional e internacional en que se gestaron tales preocupaciones ha ido creciendo en complejidad. Esto viene dado principalmente por el impacto negativo de la crisis económica mundial sobre las economías nacionales. Esta situación, unida al vertiginoso avance de la ciencia y la tecnología, incide de manera muy particular en la educación superior, pues las esferas de la información y las comunicaciones siguen avanzando a un ritmo nunca antes visto en la mayoría de los países. En consecuencia, se refuerza la necesidad de mantener la calidad de la formación profesional a un nivel que favorezca la interiorización de valores acorde con el desarrollo humano.

Para cumplir con estas demandas, el aula y el sistema de relaciones sociales que en esta se establece se identifican como el objeto de estudio. Luego, no se trata solamente de delimitar la relación sujeto-objeto de aprendizaje, sino de adentrarse en el proceso de toma de conciencia de la valoración, entre cuyos elementos constituyentes se establecen interdependencias de diversa naturaleza, en un orden de complejidad tal que reporta una cualidad resultante más profunda que la mera suma de la acción de estos elementos.

Muchos han sido los estudiosos que, a lo largo de la historia, han indagado en esa complejidad. A la luz de la diversidad de paradigmas teóricos y metodológicos en torno al lenguaje y la educación, en sus concepciones se han evidenciado propósitos muy diversos.

Así, en las primeras etapas, al seguirse un modelo proceso-producto, se trataba de identificar los rasgos personales de los docentes eficaces. Los resultados obtenidos con respecto a la relación entre características de los docentes y el aprendizaje de los estudiantes condujeron a centrar la atención en los métodos de enseñanza. A partir de los años sesenta emerge un enfoque bidireccional de la comunicación que hace centrar el análisis en las interacciones que se establecen en el aula.

Un estudio reciente reconoce que los modelos de interacción didáctica en el aula a lo largo de todo el tiempo tienen como fundamento teórico dos grandes corrientes del pensamiento educativo: "[...] la representada por el proceso-producto, vinculado al modelo de la transmisión; y las de carácter socio etnográfico lingüístico, relacionado con el modelo conversacional" (Ojalvo 123). La mencionada autora distingue una tercera tendencia, relacionada con el modelo sistémico instruccional.

- Modelo de transmisión: corresponde a la conceptualización sobre la comunicación verbal de 
Jacobson, que se desarrolla entre las décadas 30 y 50. La interacción que se estudia a partir de este modelo consiste en los procesos de codificación y decodificación del discurso del docente.

- Modelo sistémico instruccional: se refiere a propuestas de análisis, e intervención en los procesos de la escuela. Se centra fundamentalmente en favorecer los procesos cognitivos y organizacionales. Los estudiantes tienen un papel activo en su aprendizaje, pero limitado a sus funciones de receptores y transmisores de informaciones.

- Modelo conversacional: surgido a finales de la década de los setenta, supera las limitaciones anteriores, pues permite conocer y describir las lógicas de acción que se practican en el aula, la orientación de la comunicación, la influencia mutua en la construcción de significados, el proceso de negociación, así como el análisis de los procesos que forman parte de las relaciones sociales en el contexto escolar.

Por su caracterización, puede apreciarse la actualidad e interés del modelo conversacional para el tratamiento de la valoración en el aula universitaria, pues supera los modelos unilaterales de influencia educativa y permite abordar la inter y transtextualidad del discurso desde las indagaciones científicas realizadas recientemente en este campo.

En el caso del presente artículo, como se muestra en la figura 2, la reflexión de las autoras se inscribe dentro de los estudios semánticos discursivos que se desarrollan en la Facultad de Lenguas Extranjeras, a la luz de los postulados de una escuela de reconocido prestigio nacional e internacional, la establecida por el Dr. Leandro Caballero (Semántica y diccionario, "Estrategia, modalidad e ilucución...", "Modalidades semánticas del lenguaje”). Este destacado investigador cubano, después de haber realizado un profundo estudio semántico de unidades valorativas del español, conceptualiza el dicto ${ }^{3}$ como una unidad semántica comunicativamente relevante en la que se materializa un conjunto de marcas de la modalidad como supracategoría del discurso.

Esta supracategoría incluye varias macrocategorías: interés, lealtad, afectividad, certidumbre, expresividad y valoración. Esta última se considera la macrocategoría semántico modal por excelencia que expresa la posición subjetiva e intencional ante

3 Esta, según el propio autor, no solo incluye la acción -núcleo de la actividad en la vida social-, sino que la supera. lo dicho por el otro sobre la base de normas sociales que toman en cuenta el valor pragmático de los objetos y fenómenos de la realidad que clasifican en una escala axiológica.

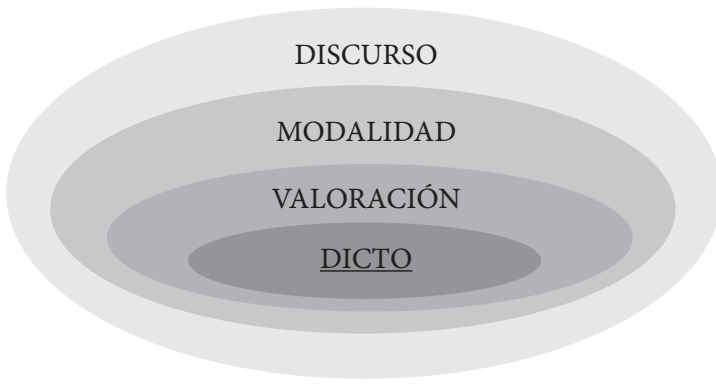

Figura 2. Estudios semántico discursivos cubanos. Fuente: elaboración propia: Llanes 2016.

Dado que los rasgos de dominio de los objetos y fenómenos valorados se realizan de manera diversa en las distintas culturas, la idea del bien se posiciona desde la perspectiva del estudiante como agente social, hablante intercultural y aprendiente autónomo en la interacción social en el grupo (Llanes). Para ampliar el encuentro entre lo subjetivo y lo cultural, que se materializa en los tipos de textos concretos, y poder precisar las manifestaciones del carácter axiológico de las valoraciones en la intertextualidad, se sigue la propuesta de Galbán de utilizar un trapecio en lugar del clásico cuadrado semiótico. En la figura 3 , se puede apreciar la ampliación de las zonas del significado que permite la figura propuesta. Al utilizar esta figura, pueden ser analizadas la ambivalencia y la ambigüedad para evadir responsabilidades ente lo dicho por el otro, lo que en la interacción puede ir cambiando, al interiorizarse nuevas informaciones, conocimientos y valores presentes en lo dicho por el otro.

Tomar el texto como objeto de estudio significa entrar en una teoría del lenguaje y en su realización en el discurso. En este sentido se postula que el texto es un todo de significaciones, un mundo de representaciones puesto en marcha a través del carácter replicativo de la palabra y realizado con enunciados relativamente estables (géneros discursivos), correspondientes a las distintas esferas de las actividades humanas (Bajtín, Marxismo y filosofía del lenguaje, Estética de la creación verbal). A partir de ello, 


\begin{tabular}{|cc|}
\hline & Ambivalencia \\
Bueno & Malo \\
Balance positivo & Balance negativo \\
No bueno & No malo \\
& Ambigüedad \\
\hline
\end{tabular}

Figura 3. Trapecio semiótico. Fuente: Galbán (30).

los rasgos pragmáticos contextuales que se deben considerar en la enseñanza de los géneros apuntan al análisis tridimensional del significado: el objeto (dimensión referencial); la intencionalidad, ilocución y estrategia (dimensión ilocutivo-modal) y la ubicación en una escala de valores lingüísticamente estructurada (dimensión constructiva-gramatical). Pues, si la gramática no está asociada a la construcción del sentido de los textos y al funcionamiento de las unidades lingüísticas en los formatos discursivos, no tiene razón de ser enseñada.

Desde la perspectiva trazada, habrá que convenir en que el proceso de enseñanza aprendizaje del español como patrimonio común de una comunidad que cada día crece más promueve una situación que debe ser asumida como nuevas posibilidades de comunicación intercultural entre representantes de diversas comunidades discursivas $y$, por ende, ha de estar presidido por un objetivo que promueva la educación continua del hombre. En ese contexto urge la necesidad de tomar en cuenta una categorización del sujeto valorador, sus intenciones, estrategias y modalidad, tres categorías imprescindibles en un enfoque intercultural del proceso de enseñanza aprendizaje.

En esto radica el interés de la autora por el tema relacionado con el tratamiento de la valoración como vía para el enriquecimiento cultural y la concienciación de la necesidad de poner límites éticos a la satisfacción de los intereses meramente personales.

\section{Diagnóstico de la situación real $\mathrm{y}$ de sus potencialidades}

En la tabla 1, se presentan los resultados de la interpretación de los datos recogidos en cinco grupos experimentales.

Tabla 1. Porcentajes por tipo de valoraciones

\begin{tabular}{lcc}
\hline \multicolumn{1}{c}{ Tipo de valoraciones } & Cantidad & Porcentaje \\
\hline Apreciativas & 215 & $84 \%$ \\
Jerarquizadoras & 28 & $11 \%$ \\
Evaluadoras & 14 & $5 \%$ \\
Totales & 357 & $100 \%$ \\
\hline
\end{tabular}

Fuente: elaboración propia.

Como se presuponía, las valoraciones apreciativas alcanzaron el mayor porcentaje. Tomando esto en consideración, en la tabla 2 , se muestran los principales indicadores que se tuvieron en cuenta para analizar las opiniones de los estudiantes.

Tabla 2. Actitud ante la idea del bien

\begin{tabular}{|c|c|c|c|}
\hline \multirow{2}{*}{ Situación } & \multicolumn{2}{|c|}{ Hechos intencionales } & \multirow{2}{*}{ Modalidad } \\
\hline & Particulares (intenciones) & Generales (estrategias) & \\
\hline $\begin{array}{l}\text { Ante un coenunciador que } \\
\text { podría estar de acuerdo } \\
\text { con la apreciación que el } \\
\text { enunciador se cree en la } \\
\text { obligación de hacer }\end{array}$ & $\begin{array}{l}\text { Expresar juicios, opinio- } \\
\text { nes positivos o negativos } \\
\text { mediante la adaptación al } \\
\text { contexto }\end{array}$ & $\begin{array}{l}\text { Valorativa: mostrar una } \\
\text { tendencia al reposo, una parti- } \\
\text { cularización y especificación en } \\
\text { relación con las normas vigen- } \\
\text { tes o aplicadas comúnmente }\end{array}$ & $\begin{array}{l}\text { Selección de pronombres personales } \\
\text { y posesivos tanto en primera como en } \\
\text { segunda persona del singular: "tú y yo } \\
\text { siempre hemos estado de acuerdo con"; } \\
\text { "mi opinión coincide con la tuya". }\end{array}$ \\
\hline $\begin{array}{l}\text { Ante un coenunciador que } \\
\text { podría no estar de acuerdo } \\
\text { con la apreciación que el } \\
\text { enunciador se cree en la } \\
\text { obligación de hacer }\end{array}$ & $\begin{array}{l}\text { Expresar juicios y argu- } \\
\text { mentos que responden } \\
\text { a valores instalados en } \\
\text { su horizonte apreciativo, } \\
\text { aunque contradictorios } \\
\text { con el coenunciador }\end{array}$ & $\begin{array}{l}\text { Manipuladora: hacer que el } \\
\text { interlocutor tolere su idea del } \\
\text { bien. } \\
\text { Explicativa: lograr el conven- } \\
\text { cimiento }\end{array}$ & $\begin{array}{l}\text { "Ustedes saben que yo soy su amigo"; "no } \\
\text { lo digo por malo" } \\
\text { "Si no fuera de su opinión, no diría esto, } \\
\text { pero lo hago porque". "Me veo en la } \\
\text { necesidad imperiosa de". } \\
\text { "Se entiende que". } \\
\text { "Se reconoce que". }\end{array}$ \\
\hline
\end{tabular}


(viene)

\begin{tabular}{|c|c|c|c|}
\hline \multirow{2}{*}{ Situación } & \multicolumn{2}{|c|}{ Hechos intencionales } & \multirow{2}{*}{ Modalidad } \\
\hline & Particulares (intenciones) & Generales (estrategias) & \\
\hline \multirow[t]{4}{*}{$\begin{array}{l}\text { Ante un coenunciador } \\
\text { evaluador }\end{array}$} & $\begin{array}{l}\text { Intensificar el grado de } \\
\text { certeza de la idea del bien. }\end{array}$ & $\begin{array}{l}\text { Explicativa: para atenuar la } \\
\text { oposición }\end{array}$ & $\begin{array}{l}\text { Se hace referencia a una alternativa. } \\
\text { Se usan recursos que abren la participa- } \\
\text { ción a los otros. } \\
\text { Incluye la probabilidad, la apariencia y el } \\
\text { rumor. Es frecuente el uso de determi- } \\
\text { nantes de conocimiento grupal: "no es } \\
\text { una opinión, la comparten"; "creo que" }\end{array}$ \\
\hline & & $\begin{array}{l}\text { Directiva: hacer evidente el } \\
\text { espacio donde se enuncia la } \\
\text { idea. }\end{array}$ & $\begin{array}{l}\text { Emplear el criterio de autoridad como } \\
\text { argumento. }\end{array}$ \\
\hline & & $\begin{array}{l}\text { Hacer evidente el prestigio del } \\
\text { enunciador. }\end{array}$ & $\begin{array}{l}\text { Se usan los recursos de inserción }{ }^{4} \text { y de } \\
\text { atribución }{ }^{5} \text {. } \\
\text { Estructuras que sirven para reforzar } \\
\text { la certeza: pronombres personales en } \\
\text { tercera persona del plural, adjetivación y } \\
\text { uso de nombres colectivos. }\end{array}$ \\
\hline & & $\begin{array}{l}\text { Identificar la comunidad del } \\
\text { autor de la cita con la del } \\
\text { coenunciador }\end{array}$ & $\begin{array}{l}\text { Se emplea una cita textual sin dar cabida } \\
\text { a otras voces y valores. } \\
\text { Se emplean fuentes reconocidas como } \\
\text { respaldo a la opinión. }\end{array}$ \\
\hline
\end{tabular}

Fuente: elaboración propia.

Estas actitudes son motivadoras en la medida en que están conectadas con el mundo real de los estudiantes. Desde esta perspectiva, el espacio de la clase adquiere otro sentido: los estudiantes son agentes sociales y hablantes interculturales que tienen que trabajar en grupo, indagar fuera del aula y, por tanto, tomar decisiones, moverse, relacionarse con otros.

El funcionamiento psicopedagógico y lingüístico en un escenario tradicional habitualmente se fundamenta en la recepción unidireccional de información en un ambiente de mucho silencio y atención. Para la valoración del sentido de la idea del bien las representaciones de la relación aprendizaje-desarrollo tienen que cambiar de perspectiva.

\section{Relación aprendizaje-desarrollo}

Como se ha ido explicitando a través de toda la conceptualización precedente y con respecto al diagnóstico, el enfoque de negociación permanente de los significados y sentidos constituye el eje de la toma de decisiones con respecto al abordaje de la diversidad de voces y valores en torno a la idea del bien, en una clase multicultural en su interdependencia dinámica y dialéctica con los diferentes componentes del proceso de enseñanza aprendizaje. Así, como se muestra en la figura 4, el diagnóstico visto como proceso que incluye no solo la situación histórico social concreta, sino también las necesidades expresadas por los estudiantes y las potencialidades que brindan las interacciones dialógicas son factores claves para establecer los objetivos, determinar los contenidos y los métodos, lo que conduce a dinamizar sentidos de vida y a romper estereotipos, aspectos esenciales para construir la idea del bien desde múltiples miradas.

\section{Conclusiones}

- El marco teórico conceptual se enfoca en la idea del bien, que incluye no solo la interacción humana para construir conocimientos, sino también como actividad en la práctica social, en la

4 Lo que comúnmente se conoce como cita directa o estilo directo.

5 Lo que comúnmente se conoce como cita indirecta o estilo indirecto. 


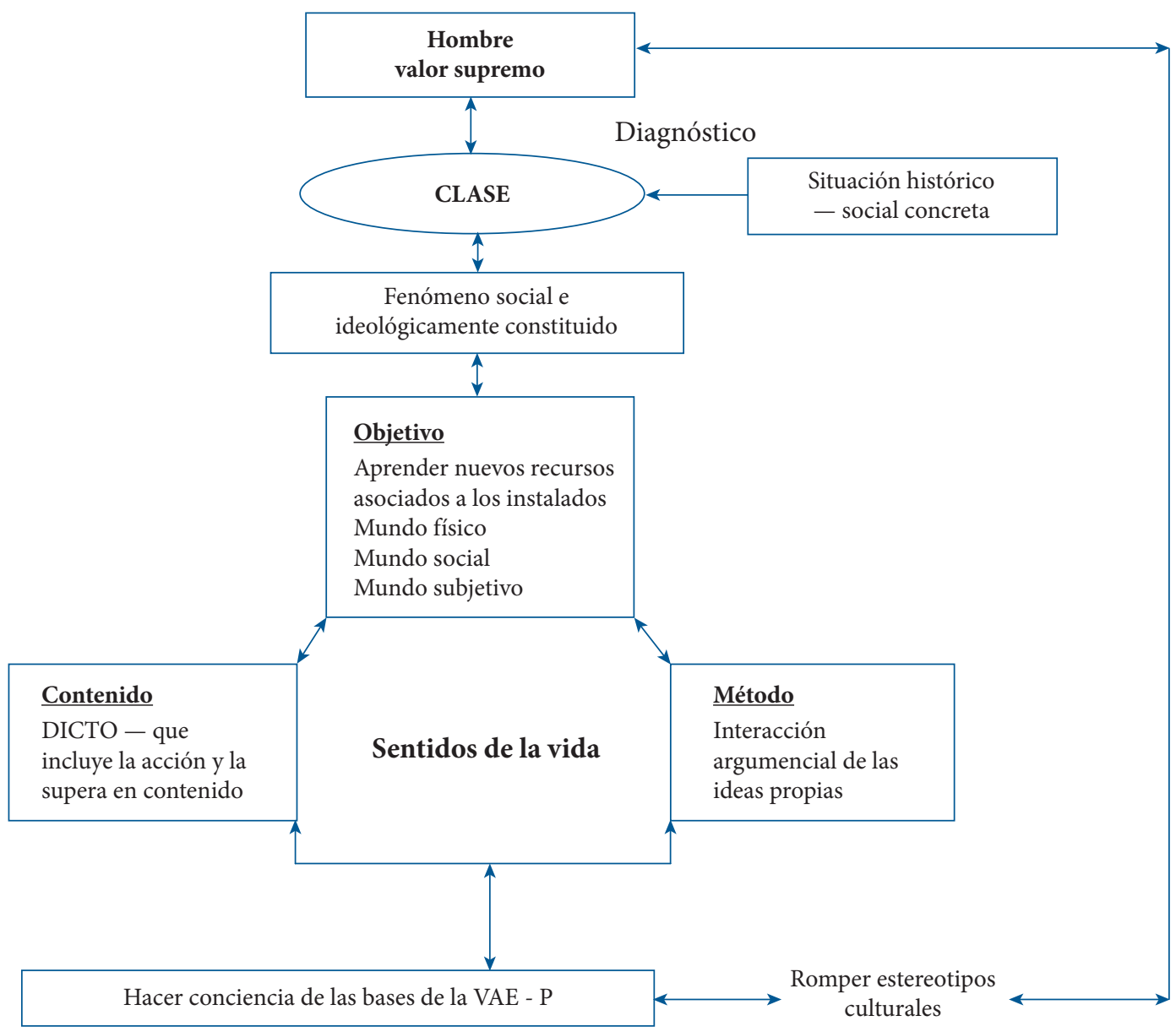

Figura 4. Relación aprendizaje-desarrollo. Fuente: elaboración propia.

cual, la apropiación, generación y uso de las valoraciones apreciativas éticas y psicológicas implican un desarrollo en el tiempo. Es decir, que la relación entre el individuo y su entorno social no puede ser reducida a una simple determinación de uno por el otro.

- El espacio de la valoración apreciativa ético y psicológico, al ser objeto de estudio específico de la linguodidáctica, interesa en la medida en que permite a los estudiantes enriquecer sus vivencias y ampliar sus conocimientos, habilidades y valores para poder comprender, reflexionar, valorar y construir la idea del bien desde múltiples miradas.

- La conceptualización del tratamiento de la valoración apreciativa ética y psicológica con base en la comprensión de lectura toma en consideración la necesidad de recuperar los objetivos formativos en la enseñanza de lenguas extranjeras, lo que implica el tratamiento de la idea del bien en la diversidad.

- El tratamiento de la idea del bien a partir de un enfoque ético psicológico permite a los estudiantes enriquecer sus vivencias, al mismo tiempo que amplía sus conocimientos, habilidades y valores para poder comprender, reflexionar y valorar desde una mayor autoconciencia.

\section{Referencias}

Acosta, Rodolfo "Enfoque interactivo para la enseñanza de lenguas”. Tesis Instituto Pedagógico “Enrique "José Varona”, 2000. Impreso.

Austin, Tomás. Para comprender el concepto de cultura. Santiago de Chile: Universidad Otero Prat, 2000. Impreso. 
Bajtín, Mijail. Marxismo y filosofía del lenguaje. Problemas fundamentales del método sociológico en la Ciencia del Lenguaje. Sao Paulo: Hucitec, 1999. Impreso.

Estética de la creación verbal. México: Siglo xxI, 1997. Impreso.

Hacia una filosofía del acto ético. De los Borradores y otros escritos. Barcelona: Anthropos, 1997. Impreso.

Beaugrande, Robert de. La saga del análisis del discurso como estructura y proceso. Barcelona: Geisa, 2000.

Caballero, Leandro. Semántica y diccionario. La Habana: Editorial de Ciencias Sociales, 2014. Impreso.

"Estrategia, modalidad e ilucución: tres conceptos de una semántica comunicativa". Estudios lingüísticos cubanos (I). Homenaje a Leandro Caballero Díaz. Valencia: Universitat de Valencia, 2002. 131-136. Impreso.

"Modalidades semánticas del lenguaje". Estudios lingüísticos cubanos (II). Homenaje a Leandro Caballero Díaz. Valencia: Universitat de Valencia, 2002. 137-160. Impreso.

Coll, César "Psicología y currículum. Una aproximación psicopedagógica al Currículum escolar". Cuadernos de Pedagogía 28 (1994): 10-20. Impreso.

Curbeira, Ana. "La interacción de las ideologías y las categorías semánticas modales en el discurso de una fábula de R. Tagore". Rastros y Rostros 15.29 (2013): 9-14. Impreso.

"Teoría y práctica del Grupo de estudios semánticos y análisis del discurso de la Facultad de Lenguas Extranjeras de la Universidad de La Habana. Anuario Instituto de Lingüistica y Literatura. Academia de Ciencias de Cuba 20 (2008): 36-39. Impreso.

Introducción a la teoría del lenguaje. La Habana: Félix Varela, 2007. Impreso.

Lecturas de semántica I. La Habana: Félix Varela, 2003. Impreso.

Dijk Van, Tuem. "Semántica del discurso e ideología”. Discurso y Sociedad 2.1: 201-261, 2008. Impreso.

El discurso como estructura y proceso: estudios sobre discurso I y II. Barcelona: Geisa, 2000. Impreso

Ideology, a Multidisciplinary Approach. London: Sage Publication, 1998. Impreso.

Diviñó, Esther. Estrategia linguodidáctica para el tratamiento de los valores a partir de la comprensión de lectura de cuentos, en condiciones de multiculturalidad (Nivel superior de los cursos de corta duración). Tesis $\mathrm{U}$. de La Habana. La Habana: Universidad de La Habana, 2016. Impreso.
Diviñó, Esther y Llanes, Rómula "Estrategia linguodidáctica para el tratamiento de los valores a partir de la comprensión de la comprensión de cuentos en la clase de E/LE y L2 en los cursos de la FENHI". Apropiación, aplicación y uso edificador del conocimiento. REDIPE - Universidad de Ciencias Pedagógicas y Editorial Académica. La Habana: Edacun, 2017. Impreso.

Fabelo, José Ramón. Los valores y sus desafíos actuales. La Habana: José Martí, 2003. Impreso

Práctica, conocimiento y valoración. La Habana: Ciencias Sociales, 1989. Impreso.

La naturaleza del reflejo valorativo de la realidad. La Habana: Instituto Cubano del Libro, 1987. Impreso.

Fique, Diana (Compiladora). Niveles de comprensión lectora: habilidades de la comunicación I. Corporación Universitaria Iberoamericana. Disponible en: https:// slideplayer.es/slide/11838223/

Galbán, Ana María. Aproximación al estudio de las macrocategorías semánticas modales (valoración, lealtad, certidumbre, interés, afectividad y expresividad) y su expresión a través de verbos de las lenguas española y alemana. Tesis U. La Habana. La Habana: Universidad de La Habana, 2003. Impreso.

Greimas, Algirdas Julius. La sémantique structurale. París: Larousse, 1965. Impreso.

Jackendoff, Ray. Foundations of Language. New York: Oxford University Press, 2002. Impreso.

Juliano, Dolores. Educación intercultural: escuela y minorías étnicas. Madrid: Universidad Complutense, 1993. Impreso.

Kristeva, Julia. Ese desconocido: el lenguaje. Sao Paulo. Fontes. 2001. Impreso

Leontiev, Alexi Nicolaievich. Actividad, conciencia y personalidad. La Habana: Pueblo y Educación, 1981. Impreso.

Lewis, Richard. When Cultures Collide: Leading across Cultures. Londres: Nicholas Brealey International, 2006. Digital.

Llanes, Rómula. Modelo didáctico para el tratamiento de la valoración apreciativa ético-psicológica en la clase de español como lengua extranjera. Tesis . U. La Habana: Universidad de La Habana, 2004. Impreso.

Llanes, Rómula. y Diviñó, Esther. "Diversidad de voces y valores: nuevas posibilidades de comunicación”. Apropiación, aplicación y uso edificador del conocimiento. Redipe. Universidad de Ciencias Pedagógicas y Editorial Académica. La Habana: Edacun, 2016. Impreso.

"La valoración como indicador de los cambios en el aula universitaria". Memorias de la X Conferencia In- 
ternacional Lingüística, 2017. La Habana: Instituto de Literatura y Lingüística, 2017. Digital.

Llanes, Rómula y Nicolau, María del Loreto. "La valoración apreciativa ético-psicológica en el aula universitaria ante los procesos de cambio". Ecociencia 4.3 (2016):17 -24. ECOTEC. Impreso.

Ojalvo, Victoria. "Las interacciones en el aula: un fructífero campo de investigación educativa”. Revista Cubana de Educación Superior, 0257.4314(2012): 121- 138. Impreso.

Sánchez, Aquilino. Hacia un método integral de la enseñanza de idiomas. Madrid: SGEL, 2009.
Vygotsky, Liev. Simionalich. Pensamiento y lenguaje. Teoría del desarrollo de las funciones psíquicas. La Habana: Pueblo y Educación, 1999 a. Impreso.

La formación Social de la Mente. Caracas: Monte Ávila, 1999 b. Impreso.

Wong, Ernesto Primer acercamiento al estudio de la macro-categoría semántica modal de interés en la lengua española. La Habana: Memorias de la Conferencia Internacional Lingüística 2017. Digital.

"Un modelo para el análisis semántico discursivo de la causalidad”. Tesis U. de La Habana, 2015. Inédito. 